\title{
An Improved infrared and visible image fusion algorithm based on the Second Generation Curvelet Transform
}

\author{
Liu Xiaojun \\ School of Electronic \& Information \\ Huanggang Normal University \\ Hubei Huanggang, China \\ whutliuxiaojun@126.com
}

\author{
Wang Jianyu \\ School of Transportation \\ Huanggang Normal University \\ Hubei Huanggang, China \\ whutliuxiaojun@126.com
}

\begin{abstract}
The fusion of infrared images and visible images can combine complementary information in an image, so we can better describe a scene, and it is helpful for some tasks such as target detection, target localization and environment recognition. In this paper, we use the Second Generation Curvelet Transform (SGCT) to decompose infrared images and grayscale visible images to propose a new image fusion algorithm. This algorithm uses a multi-resolution decomposition of different tools and different fusion rules implementation. The simulation results show that, compared with existing algorithms, this algorithm have improved to some extent in the evaluation of fused images.
\end{abstract}

Keywords- Image Fusion; Multi-resolution Analysis; the Second Generation Curvelet Transform

\section{INTRODUCTION}

Wavelet Transform (WT) has the localized analysis capabilities of time domain and frequency domain, as well as its optimal approximation performance in onedimensional bounded function, making WT an important tool for analyzing and processing one-dimensional nonstationary signals $^{1]}$. However, WT cannot make full use of the geometric regularity of image itself. In order to overcome the deficiencies in the image analysis of WT, EJ Candès and DL Donoho ${ }^{[2]}$ proposed Curvelet transform. Curvelet transform has the property of anisotropy, but its mathematical realization is more complex in its transformation process. In view of this, EJCandes and DL Donoho proposed a simpler, more easily understood Curvelet theory in 2004, and it is known as the Second Generation Curvelet Transform (SGCT) ${ }^{[3-4]}$.

Curvelet transform as a new MRA tool ${ }^{[5-7]}$, compared with WT, is it is more suitable for portraying images of geometric features such as curves and lines, using the " wedge base" to approach the singular point of $C^{2}$, and fully consider the geometry of the singular point, and with any angle of directionality (anisotropy), so it is more suitable for the image processing and application. In addition, Curvelet transform has a better factor expression ability in the geometric characteristics of the images (curves, straight lines), they can be expressed through a small number of large Curvelet transform coefficients, and more energy concentrated after the transformation, so it is of great importance in the image extraction and analysis.

\section{INFRARED AND VISIBLE IMAGE FUSION ALGORITHM BASED ON THE SECOND GENERATION CURVELET TRANSFORM (SGCT)}

The characteristics of infrared image are lower space resolution, serious mixing phenomenon, easy to lose high frequency information details, while the visible image features opposite, other than less low frequency information ${ }^{8-9]}$. Thus, the fusion of infrared images and visible images has been widely applied to obtain complementary image information, with which we can better describe a scene and complete some tasks, such as target detection and localization, environment identification $^{[10-11]}$.

\section{A. Steps and framework}

In this paper, decompose the infrared and gray visible images with SGCT and propose the image fusion algorithm based on significance level and regional matching rule. Steps are as follows:

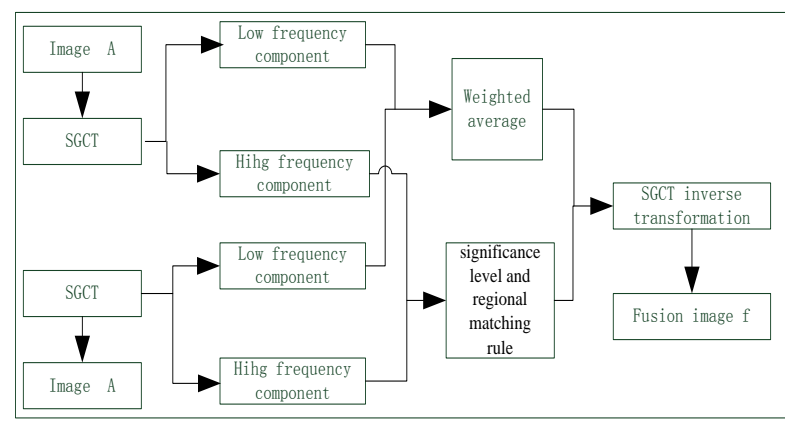

Figure 1. The framework of infrared and gray visible image fusion algorithm based on SGCT

1. Convert infrared image A and visible image B to SGCT respectively, then obtain their low frequency and high frequency components;

2. Process the above low and high frequency components with their own fusion strategies to obtain the fused components;

3. Process the fused components with SGCT Inverse Transform to obtain the final fusion 
images. Specific framework of the fusion images is shown in Fig.1:

\section{B. Realization process of fusion algorithm.}

Image fusion rule determines the ultimate fusion effect directly, which is the core of fusion algorithm and also a key issue not solved effectively so far.

1) Directional contrast.

The concepts of directional contrast and weighted absolute values are proposed in literature ${ }^{[12]}$ and literature ${ }^{[13]}$ in order to measure the images on different scales and directional visual brightness. In SGCT, the image can be decomposed into low and high frequency sub-band, and the directional contrast ${ }^{[13]}$ is defined as follows (1): Among them: $\mathrm{X}=(\mathrm{A}, \mathrm{B})$.

$$
R^{X}(x, y)=\frac{H_{j}^{X}(x, y)}{L_{j_{0}}^{X}(x, y)}
$$

\section{2) Weighted activity}

Weighted activity can be used to measure the information quality of a pixel point and represent contained information content. The research based on human visual system points out that the human eyes are sensitive to the areas changed dramatically such as edge and contour information, where these areas usually contain more pixels and include more details than any other smoother areas. Weighted activity is defined as expression (2), which is calculated in a S'T smooth window.

$$
C_{j}(x, y)=\left|H_{j}(x, y)-W(p, q) H_{j}(x+p, y+q)\right|
$$

Among them, $\mathrm{W}(\mathrm{P}, \mathrm{Q})$ is an activity weight, changing with correlation of the pixel $(x+p, y+p)$ and the center pixel (x, y).

\section{3) Significant level measure}

Significant level measure reflects some significant features of multi-resolution coefficients of the source images. Regard the above defined directional contrast and weighted activity as significant level measure, then the highest SGCT decomposition level of significant level measure can be defined as follows (3):

$$
A_{L}^{X}(x, y)=R_{L}^{X}(x, y)
$$
(4):

For other decomposition level, definition is as follows

$$
A_{j}^{X}(x, y)=R_{j}^{X}(x, y)
$$

Among them, $X=(A, B), j=1,2 \ldots L-1, L$ is decomposition level.

Use matching measure to represent the similarity degree of multi-resolution coefficients between the two source images, and is defined as follows(5):

$$
M_{j}(x, y)=\left|\frac{\operatorname{Min}\left(A_{j}^{A}(x, y), A_{j}^{B}(x, y)\right)}{\operatorname{Max}\left(A_{j}^{A}(x, y), A_{j}^{B}(x, y)\right)}\right|
$$

Among them, $X=(A, B), j=1,2 \ldots L-1, L$. High matching measure indicates more similarities between source images.

\section{4) Fusion algorithm}

Fusion algorithm based on weighted average method is shown as follows (6):

$$
L_{j}^{F}(x, y)=\frac{L_{j}^{A}(x, y)}{L_{j}^{A}(x, y)+L_{j}^{B}(x, y)} L_{j}^{A}(x, y)+\frac{L_{j}^{B}(x, y)}{L_{j}^{A}(x, y)+L_{j}^{B}(x, y)} L_{j}^{B}(x, y)
$$

Among them, $L_{j}^{A}(x, y)$ and $L_{j}^{b}(x, y)$ represent the value of the point $(\mathrm{x}, \mathrm{y})$, and $L_{j}^{F}(x, y)$ is the fused value of the same point. According to the fusion rule, process the fused components with SGCT inverse transform to obtain fusion image $\mathrm{F}$.

\section{SiMULATION EXPERIMENTS AND RESULTS ANALYSIS}

The experiment selects two groups of infrared and visible light image to simulation, which used to compare to verify the effectiveness and correctness of proposed algorithm . The first group of source images are infrared and visible light images in the investigation which is shoot at the same scene. In 2 (a)), infrared image can clearly distinguish a standing person, compared with hot objects in the scene ,others are of low contrast, fuzzy objects, difficult to distinguish, and the same scene visible light image (Figure 2 (b)), roads, fences, roof, chimney scene contrast is high, but because of the dim light, the figure can not be seen.

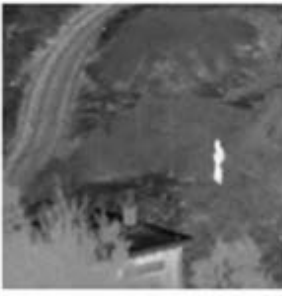

(a) infrared image

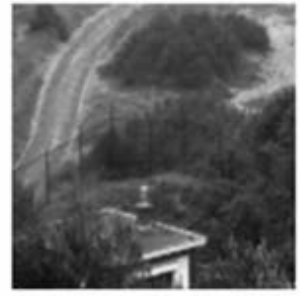

(b) visible image
Figure 2. The source images (a)infrared image (b)visable image

After SGCT decomposition. Infrared, visible, and the fusion of various scale add up the results as shown in figure 3 (a), (b), (c), which shows the result of the infrared, visible and fusion of various scale additive for edge detection. From the above experimental performance analysis, SGCT can more sparse representation of the source image of geometric features such as curve, linear and so on, through a few larger Curvelet transform coefficient can be said, and after the transformation energy is more concentrated, which is of great significance for extraction and analysis of image characteristics. Fusion algorithm based on SGCT and the significant measure to 
combine features of the image of the infrared and visible light image, effectively retain the image characteristics of ground objects in the scene (background) information and hot target (Figure)information, which is marginal as well as clearly distinguishable.

In order to verify the validity and correctness of fusion algorithms in this chapter, experiments are compared with 3 kinds of typical fusion algorithm, the first is based on the LPT image fusion algorithm, decomposition of 5 layer, and the top LPT sub-band coefficients of fusion with weighted average method; the second is the fusion algorithm of image based on DWT, using the "DB4" wavelet filters, and image fusion image decomposition algorithm for 5 level; the third is based on the SGCT image fusion algorithm SGCT simple),decomposition of 5layer.To able comparability between the fusion algorithm, with the fusion processing, the second and third kinds of fusion algorithm based on low frequency coefficient the weighted average, high frequency coefficient of regional energy from fusion rule. Figure 4 (a), (b) gives the first set of infrared and visible light images to be fused, (c) (f) is a variety of the fusion results.

From the visual point of view, in figure 4, the fusion algorithm can heat the target in infrared image (figure) characteristics and location characteristics combined with visible light image characteristics of background information. From the perspective of the observations of the target, as shown in the red line marking region in figure 4, Through the comparison we can find that figure 4 (d) has obvious "block" effect, the characters are virtual shadow edge, which is owing to the lack of the translation invariance for DWT, thus there has produced many irregular wave and edge in the reconstructed image. By comparison, because of the SGCT excellent directional characteristic as well as the better recognition ability of describing the edge details of image feature,in figure 4 (e), (f), the phenomenon of the "block" effect and the virtual shadow can be eliminated.

In order to evaluate different fusion algorithm for infrared and visible image fusion performance, fully integrated into the source image of the infrared target characteristics and background edge detail information, this paper uses entropy $(\mathrm{H})$, correlation coefficient (CC), average gradient (G), space frequency (SF) as an objective evaluation criteria. Results as shown in table 1.

From the evaluation of fusion results can be seen, compared with other algorithm in simulation experiments, the proposed algorithm for performance evaluation indexes all have a certain degree of increase. Entropy algorithm in this paper, and the correlation coefficient are higher than other three algorithms, which shows the fusion algorithm proposed in this paper have more important feature information in the original image. The average gradient and spatial frequency of this algorithm are significantly higher than other algorithm, which shows that this algorithm keep in visible light image feature information and the background of infrared image of target feature information, at the same time, it can effectively keep the edge details of the source image features, which exactly the same as that of the subjective visual comparison results.

\section{CONCLUSION}

Based on the multi-resolution analysis of image fusion framework: multi-resolution analysis algorithm is the basis of image fusion, the fusion rule is the core of image fusion, the fusion effect evaluation is the key of image fusion. In view of this, this paper introduces the second generation Curvelet transform multi-resolution tools to image fusion , which combines with human visual characteristics and multi sensor imaging characteristics of prior information, so a new algorithm is proposed, and the algorithm is applied to the infrared and gray level image fusion. Through the simulation experiment, the effectiveness of the algorithm is verified.

\section{ACKNOWLEDGEMENTS}

The project was supported by the Doctoral Scientific Fund Project of Huanggang Normal University (Grant No. 2013031103), and the Scientific Research Program of Educational Commission of Hubei Province of China (Grant No. 20142905).

\section{REFERENCES}

[1] Shi W, Zhu C, Tian Y, et al. Wavelet-based image fusion and quality assessment[J]. International Journal of Applied Earth Observation and Geoinformation, 2005, 6(3): 241-251P.

[2] E. J. Candes, Donoho D. L. Curvelets: a surprisingly effectively non-adaptive representation for objects with edges[J]. Curve and Surface Fitting. Saint-Malo, 1999, 1- 10P.

[3] E. J. Candes, Donoho D. L. Ying L. Fast discrete curvelet transforms[J]. Applied and Computational Mathematics, California Institute of Technology, 2005, 74-91P

[4] E. J. Candes, L. Demanet, D. L. Donoho. Fast Discrete Curvelet Transform[J]. SIAM Multiscale Model, Simul. 2006, 34-39P

[5] E.J. Candes, D. L. Donoho. Curvelets A suprisingly effective non adaptive representation for objects with ddges[J]. Vanderbit University Press, Nashvile, TN, 2000.

[6] J. L. Starck, E. J. Candes and D. L. Donoho. The Curvele Tansform for Image Denosing[J]. IEEE Tran. on Image Processing, 2002, 11(1): 670-684P.

[7] E. J. Candes, D. L. Donoho. Curvelets, Multiresolution Representatin and Scaling Laws[J]. SPIE Wavelet Applications in Signal and Image Processing, 2001, 223-236P

[8] S. Yin, L. Cao, Y. Ling. One color contrast enhanced infrared and visible image fusion method[J]. Infrared Physics and Technology, 2010, (53): 146-150P

[9] Y. Lanir. Comparing multispectral image fusion methods for a target detection task[M]. Master dissertation, Dept. Indus. Eng Manage. Ben-Gurion University of the Negev,2005.

[10] Zhao Jingchao, Qu Shiru. A better algorithm for fusion of infrared and visible image based on curvelet transform and adaptive pulse coupled neural networks[J]. Journal of Northwestern Polytechnical University, 2011, 29(6): 849-853P.

[11] A. Toet, E. M. Franken, Perceptual evaluation of different image fusion schemes[J].Displays, 2003, 25-37P.

[12] Tian Pu, Qingzhe Fang, Guoqiang Ni. Contrast-based multiresolution image fusion[J].Acta Electronica Sinica. 2010, 28(12): 116-118P.

[13] Yifan Zhang, Mingyi He. Absolute value activity and regional similarity based on image fusion[J]. Computer Engineering and Application. 2006(18): 8-10P. 


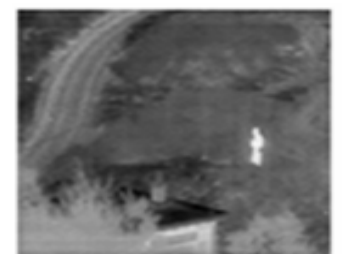

(a) sum of infrared all scales

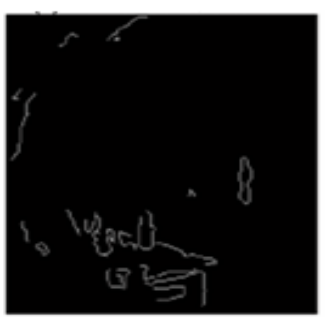

(d) edge area of infrared

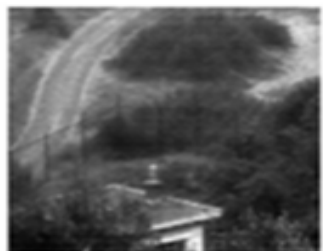

(b) sum of visible all scales

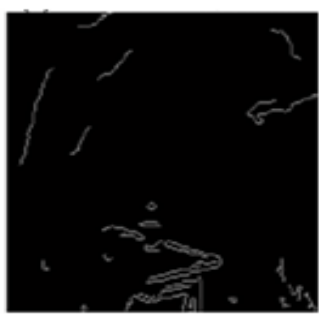

(e) edge are a of visible

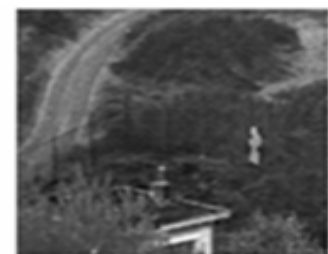

(c) sum of fusion all scales;

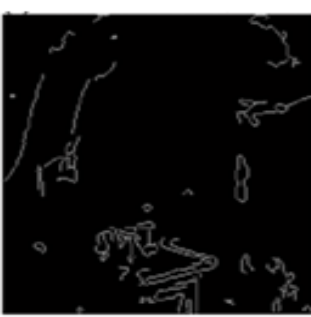

(f)edge area of fusion result

Figure 3. The sum of all scales results and detected areas (a) sum of infrared all scales; (b) sum of visible all scales; (c) sum of fusion all scales; (d) edge area of infrared; (e) edge area of visible; (f) edge area of fusion result

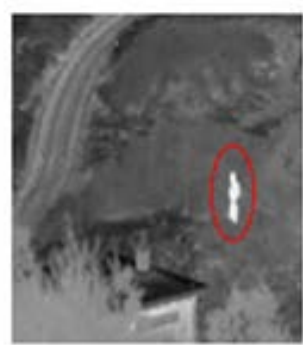

(a) infrared image

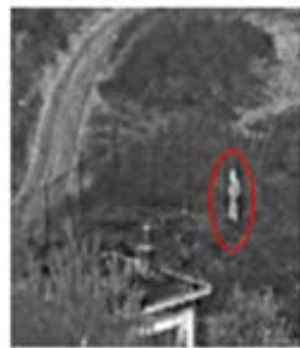

(d) DWT fusion

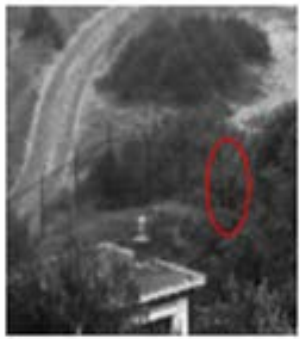

(b) visible image

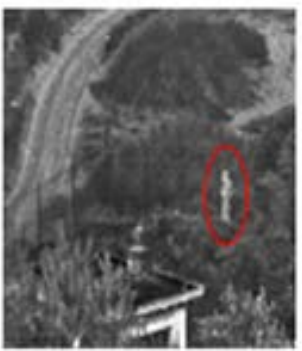

(e) SGCT_simple fusion

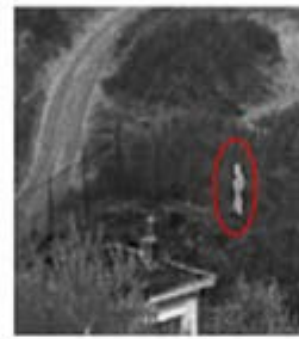

(c) LPT fusion;

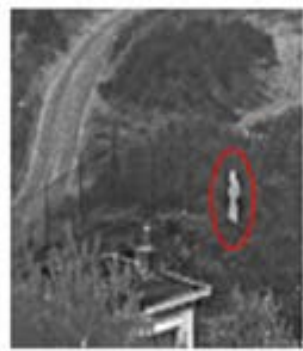

(f) proposed fusion

Figure 4. The experiment of image fusion of group1(a) infrared image; (b) visible image; (c) LPT fusion; (d) DWT fusion;(e) SGCT simple fusion; (f) proposed fusion

TABLE I. EVALUATION OF THE FUSION ALGORITHM

\begin{tabular}{|c|c|c|c|c|}
\hline \multirow{2}{*}{ Fusion method } & \multicolumn{4}{|c|}{ Evaluation indicators } \\
\cline { 2 - 5 } & $\mathrm{H}$ & $\mathrm{CC}$ & $\mathrm{G}$ & $\mathrm{SF}$ \\
\hline LPT & 7.0783 & 0.7524 & 8.9022 & 12.3238 \\
\hline DWT & 7.1539 & 0.8146 & 9.1842 & 12.5198 \\
\hline SGCT & 7.1976 & 0.8595 & 9.2103 & 12.8058 \\
\hline This Paper & 7.2598 & 0.9066 & 9.2542 & 13.0956 \\
\hline
\end{tabular}

